\title{
Procedimientos de diseño en mecatrónica
}

\section{Procedures of Design in Mechatronics}

\author{
González-Palacios M.A. \\ Departamento de Ingeniería Mecánica \\ Universidad de Guanajuato \\ E-mail:maxg@salamanca.ugto.mx
}

Información del artículo: recibido: noviembre de 2008, reevaluado: febrero de 2010, aceptado: septiembre de 2010

\section{Resumen}

En este documento se presentan los conceptos fundamentales relacionados con los procedimientos del diseño en mecatrónica. En una forma sistemática se desarrolla un caso de estudio consistente en el diseño de una máquina de tecnología de punta. De esta manera, se plantean los criterios más importantes que se deben tomar en cuenta en todo el proceso. Así se establece que el punto de partida es el planteamiento del problema a través de ideas, las cuales se plasman en una serie de bocetos que servirán de base para definir y decidir por la solución más factible. Se hace hincapié que desde el momento de trazar los bocetos se deben tener muy claras las dificultades a las que el diseñador estará expuesto, y que la mejor manera de enfrentar este problema es contar con una formación teórico-práctica integral. Con todo esto, se establece que la generación de prototipos virtuales representa una etapa muy importante, pues se simulan los movimientos y se pueden detectar posibles conflictos antes de pasar a procesos de manufactura de partes. También se enfatiza que el control sincronizado de los movimientos de una máquina debe integrarse tanto mecánica como electrónicamente a través de un diseño adecuado de software. Al conjugar todos los conceptos analizados en este documento, se incrementan las probabilidades de éxito en la creación de un dispositivo innovador.

\footnotetext{
Abstract

This document presents fundamental concepts related to the procedures of design in Mechatronics. It is developed systematically a case study which consists in the design of a state of the art machine. This way, the most important criteria to be considered in the design process are established. Then, it is settled down that the departing point is the problem planning through brain storm ideas, which are then drafted into sketches to provide enough information to help in the decision of the best feasible solution. It is remarked that from this stage, it is important to take into account all difficulties the designer faces, and the best way to attack this problem is the formal theoretical and practical integral training. With all this, it is stated that virtual
}

\section{Descriptores}

- prototipo virtual

- automatización

- diseño, control

- reconocimiento de voz

- interfaz gráfica

\section{Keywords}

- virtual prototype

- automation

- design, control

- voice recognition

- graphical interface 
prototyping with the aid of computer graphics represents a very important stage since the moving parts are simulated and possible conflicts or collisions can be detected before starting the manufacture of the parts. It is also emphasized that the synchronized motion control of a machine must be integrated not only mechanically, but also electronically through a suitable software design. Once all the concepts analyzed in this document are combined, there are high expectations of success when creating a state of the art machine.

\section{Introducción}

Para ilustrar los procedimientos de diseño en mecatrónica desde su fase conceptual hasta la realización de un sofisticado sistema automatizado, se presenta el siguiente caso de estudio que consiste en el diseño de una máquina sorteadora, la cual se define como Horizontal Sorting System, que en lo sucesivo se referirá a ésta como la HSS. De esta manera y para proporcionar una imagen clara de este caso de estudio, en la primera sección se describe en forma breve el proceso, posteriormente, se presenta el objetivo fundamental incluyendo las características principales que la HSS debe cubrir. En la siguiente sección denominada Conceptualización, se hace hincapié en la importancia de tener una formación integral de las disciplinas involucradas en el diseño que se esté desarrollando, además, en esta sección se plantea también la importancia de la generación de prototipos virtuales como una fase preliminar a la creación del prototipo real. Después, en la sección de Integración se introducen los conceptos de identificación de partes y sub-ensambles para auxiliar en el mantenimiento del proceso del diseño cuando se van presentado modificaciones y adecuaciones en la constante tendencia a la perfección y mejoramiento de producto deseado. En la siguiente sección, El diseño de las partes, presenta el proceso de cómo se diseñan a detalle cada una de las partes que integran los sub-ensambles, particularizando en uno de los sub-ensambles de la HSS. Se dedica la siguiente sección a la discusión de los aspectos relacionados con el software de control y sincronización de todas las partes movibles para que se lleve a cabo la tarea que se definió en el objetivo. Finalmente, en la sección Eficiencia, se discute brevemente sobre los imprevistos y de cómo éstos pueden afectar en el desempeño y la eficiencia global del producto diseñado.

\section{Planteamiento y objetivo}

Suponga que se tienen que inspeccionar láminas (hojas) de chapa de madera cuya área es de 1200 X 2400 mm con un espesor promedio de $0.7 \mathrm{~mm}$ y un peso aproximado de $1.5 \mathrm{Kg}$, a su vez, estas hojas han sido formadas mediante la unión de franjas de un ancho específico, pero que puede variar entre 60 y $300 \mathrm{~mm}$ por la misma longitud de $2400 \mathrm{~mm}$. La inspección se fundamenta en observar la hoja para determinar su calidad. Esta última depende de varios factores, los principales son la veta, el grano y la perfección de las uniones. De esta manera, dependiendo de la calidad de la hoja se tendrá que decidir a qué pila en formación será llevada. Puede darse el caso de que se tengan hasta 10 opciones. Cualquier pila que acumule un determinado número de hojas de la misma calidad es removida de la fila y queda el espacio para iniciar una nueva pila. El operador toma hoja por hoja de la pila de inspección y las desliza hasta su destino. En promedio, esta tarea le lleva quince segundos, de los cuales, entre cinco y ocho segundos son necesarios para determinar la calidad por parte de un operador calificado. No es difícil deducir que en una jornada de 8 horas al $90 \%$ de eficiencia, el operador puede clasificar hasta 1700 hojas, y que para lograr este objetivo tiene que caminar en forma intermitente alrededor de $10 \mathrm{~km}$.

Cuando el ingeniero se da a la tarea de resolver un problema de diseño, debe tener claramente definido el objetivo, que en este caso se plantea como sigue:

Diseñar una máquina que elimine al máximo el desgaste físico del operador y que su capacidad de producción dependa solamente del tiempo que le lleve al operador decidir por la calidad de la lámina.

Además, es importante estipular las características principales que la máquina debe cubrir:

Confiable. El manejo de la hoja debe hacerse cuidadosamente para evitar cualquier daño y no demeritar su calidad.

Modular. Debe tomarse en cuenta que el diseño sea válido, tanto para una máquina de dos estaciones como de más de diez.

Flexible. El software de control debe tener la capacidad de programarse de acuerdo a los diversos tipos de madera del que están hechas las hojas que serán sorteadas, ya que en este caso el número de las calidades varía de acuerdo al tipo.

Alto rendimiento. Debe tener la capacidad de trabajar continuamente y sin interrupciones.

Bajo costo. En el proceso del diseño se determinan varias soluciones que cumplen con los requisitos; $\sin$ 
embargo, el factor costo ayudará a delimitar las opciones.

Cuando se tiene definido el objetivo junto con las características y/o requisitos mínimos a cumplir, es importante hacer una búsqueda de maquinaria que se haya desarrollado en el mercado que mejor cumpla las especificaciones, de manera que se pueda partir de una base o concepto ya desarrollado. En particular, no se encontró algún equipo que cumpliera plenamente los requisitos y aquellos que mejor se acercaban no se consideraron viables de tomar como base, ya que éstos equipos fueron diseñados para transportar hojas de espesores mayores a $3 \mathrm{~mm}$ y sus sistemas de transporte desempeñan velocidades mayores a las que las hojas menores a $1 \mathrm{~mm}$ de espesor pueden soportar sin dañarse o afectar su calidad. En este caso de estudio, se tomó la decisión de diseñar la máquina partiendo de cero.

\section{Conceptualización}

Como ya se mencionó anteriormente, a un operador calificado le toma entre cinco y ocho segundos definir la calidad de la hoja, por lo que se tomó como base una capacidad entre 6 y 8 hojas por minuto. La conceptualización es una de las etapas más interesantes, porque el diseñador pone en juego toda su imaginación y plasma sus ideas en múltiples bocetos. Además, durante esta etapa, es importante conocer la opinión de los interesados, que en este caso son los operadores calificados que realizan esta tarea manualmente.

Aunque sólo se dibujen esquemas, es importante tener siempre en mente la factibilidad de fabricación de los componentes. Por consiguiente, las probabilidades de éxito dependen considerablemente por una parte, de la experiencia de haberse familiarizado previamente con partes mecánicas, y por otra, del conocimiento teórico de las propiedades físicas de las mismas. Es por esto que el buen diseñador debe pasar por un proceso de formación teórico-práctico integral.

Después de discutir varios conceptos se debe optar por el que mejor cumpla los objetivos y las restricciones. En este caso se decidió por tener una línea de transporte por bandas con estaciones por debajo para formar las pilas. Cada estación conteniendo un dispositivo para tomar la hoja de la línea y apilarla. La figura 1 muestra una copia del boceto original que representa dicho concepto, a partir del cual se desprendió el desarrollo de la HSS. En este boceto se indican dos vistas parciales, a saber, lateral y de planta.

Habiendo elegido el boceto que representa un fuerte potencial en la solución del problema, se procede a hacer uso de una herramienta de dibujo asistido por computadora (Omura, 2009), de preferencia que cuente con la capacidad de diseñar en forma paramétrica, de manera que se simplifique el proceso de modificar dimensiones cuando sea necesario. En la actualidad, los avances tecnológicos de la computación gráfica han permitido que sea posible diseñar a detalle modelos sólidos de cada una de las partes de que se compone una máquina o prototipo y así probar que los ensambles entre piezas que se conectan mutuamente, se logren conforme a los requerimientos. De esta manera, al conjunto de partes ensambladas que realizan una función definida se denomina sub-ensamble (sistema), a su vez, el conjunto de sub-ensambles conformará la máquina o prototipo virtual.

Cuando se diseña el prototipo virtual, es importante tener presentes una serie de premisas que permitirán acotar los parámetros involucrados en el diseño. A continuación se enlistan las que se consideraron de prioridad en este caso de estudio:

- Definir las limitaciones del espacio físico.

- Diferenciar las cargas estáticas de las dinámicas y establecer con claridad aquéllas a las que los subsistemas o partes mecánicas estarán sometidos.

- Encontrar patrones de movimiento que puedan convertirse con facilidad en modelos.

- Relacionar en lo posible las partes con aquellas que se pueden localizar en el mercado industrial. Aquí se listan algunas de las más relevantes marcas que se utilizaron en este proyecto: (BDI, 2007), (Fabory Masters, 2009), (Igus, 2007), (IPS Parker, 2007), (KB Electronics, 2007), (Kontron, 2010), (Morse, 2002), (NTN, 2006), (Omron, 2006), (Panduit, 1998), (Plastique Polyfab Inc., 1998), (Rittal, 2008), (SMC, 2000),

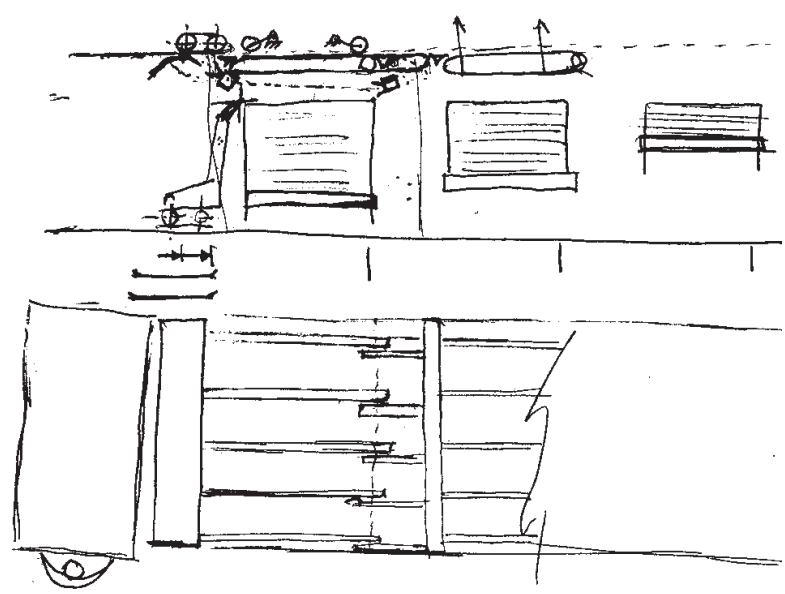

Figura 1. Boceto seleccionado para el diseño 
(Turck, 1995), (U.S. Electrical Motors, 1995), (Wieland, 1999), (Winsmith, 1997).

- Establecer los elementos que es necesario dibujar con detalle para su fabricación.

- Considerar probables fuentes de energía, tales como, neumáticas, hidráulicas, eléctricas, térmicas, etc.

- Identificar los dispositivos, mecanismos y actuadores que pueden estar involucrados en el movimiento.

- Establecer la lógica de los movimientos y sus controles.

- Implementar algoritmos y/o diagramas de flujo que ilustren la lógica del sistema.

- Desarrollar el software necesario para controlar los movimientos de la máquina a través de microcontroladores.

- Diseñar bancos de prueba para experimentar parcialmente procesos que son parte integral del dispositivo.

Cabe mencionar, que adicionalmente, se deben tomar en cuenta normas y estándares establecidos por asociaciones reconocidas a nivel nacional o internacional, según sea el alcance que se le quiera dar al diseño.

Actualmente, el internet proporciona una gran ventana de acceso a la información y se ha convertido en una herramienta poderosa de consulta; sin embargo, es conveniente contar además, con manuales especializados que proporcionan una asistencia constante en el proceso del diseño, en particular, el diseño de maquinaria (Oberg et al., 2008).

Una vez que se completa el prototipo virtual satisfactoriamente, se procede a generar todos los dibujos de fabricación a partir del modelo sólido de cada parte, teniendo así la certeza de que el prototipo real se ensamblará libre de errores, siempre y cuando las partes cumplan con las tolerancias especificadas.

\section{La integración del diseño}

A medida que se va integrando el diseño, primero las partes que comprenden un sub-ensamble y posteriormente los sub-ensambles que comprenden la máquina, van surgiendo necesidades de modificar partes o incluso de modificar sub-ensambles completos. Es posible llevar un registro de la evolución del diseño administrando los archivos de los dibujos de forma sistemática. Así, en la tabla 1 se muestra la simbología que permite codificar cada parte de una máquina. En caso de existir sub-ensambles de un sub-ensamble o partes de un sub-sub-ensamble, se pueden identificar agregando campos posteriores al de SSS. En el caso de la HSS sólo existen sub-ensambles y sus partes correspondientes. Como criterio general aunque puede variar el número, se reser- van tres caracteres para identificar nombres de ensambles y dos para identificar números de parte o de revisión. Para la HSS, después de varias iteraciones se llegó a la siguiente descripción del concepto. La línea estará compuesta de estaciones o módulos. Cada módulo contará con un sistema de transporte por banda, el cual será abatible para entregar la hoja al siguiente módulo; un sistema de sujeción instalado por debajo del sistema de transporte para recibir la hoja del módulo anterior y jalarla hasta su posición de apilamiento; un sistema de apilamiento de altura constante; un sistema estructural que soportará los demás sistemas del módulo. Esta conceptualización no se aleja mucho de la propuesta en la figura 1. Sin embargo, hubo cambios que mejoraron el concepto al minimizar costos y mejorar el desempeño. Entre los cambios más representativos, se puede mencionar que el sistema de transporte originalmente se desplazaría horizontalmente hacia arriba, lo que implicaba cargar con todo el peso del sistema, mientras que en la solución final un extremo es articulado. Otro cambio fue que originalmente se sugirieron rodillos de arrastre en cada módulo, los cuales fueron eliminados, excepto en el primer módulo. En la tabla 2 se muestran los sistemas arriba mencionados y su codificación correspondiente. En esta tabla se presentan también los

Tabla 1.Códigos de identificación de dibujos

\begin{tabular}{lll}
\hline & \multicolumn{1}{c}{ Concepto } & \multicolumn{1}{c}{ Código } \\
\hline & \multicolumn{1}{c}{ Ensamble } & 0-EEE-nn \\
& \multicolumn{1}{c}{ Subensamble } & 0-EEE-SSS-nn \\
& \multicolumn{1}{c}{ Parte } & \multicolumn{1}{c}{ EEE-SSS-pp-nn } \\
0: & identificador entre partes y ensambles \\
EEE: & caracteres identificadores del nombre de la máquina o \\
& proyecto \\
SSS: & $\begin{array}{l}\text { caracteres reservados para identificar el nombre del } \\
\text { subensamble }\end{array}$ \\
pp: & número de parte. \\
nn: & número de la revisión & \\
\hline
\end{tabular}

Tabla 2. Subensambles de 0-HSS

\begin{tabular}{lc}
\hline \multicolumn{1}{c}{ Nombre del sub-ensamble o sistema } & Código \\
\hline Estructura o soporte & 0-HSS-FRA \\
Plataforma desplazable verticalmente & 0-HSS-BAS \\
Transportador de banda & 0-HSS-BED \\
Brazo sujetador & 0 -HSS-GRI \\
Consola principal & $0-$ HSS-CP0 \\
Consola secundaria & $0-H S S-C P 1$ \\
Gabinete satélite & $0-H S S-C P 2$ \\
Sistema Hidráulico & 0 -HSS-HYD \\
Sistema Neumático & $0-H S S-P N E$ \\
\hline
\end{tabular}


sub-ensambles relacionados con las instalaciones eléctrica (CP0 y CP1), hidráulica (HYD) y neumática (PNE).

Como se indicó anteriormente, el diseño de todo el sistema debe ser modular, por lo que cada estación de apilamiento es independiente. No obstante, al unir los módulos se forma una sola línea, en particular, la primera línea que se puso en marcha es de diez estaciones. El sub-ensamble de la estructura que soporta cada módulo se denomina 0-HSS-FRA y la figura 2 muestra tres vistas con dimensiones globales en mm. También se pueden observar detalles de las bisagras de las puertas de acceso en las que se integra un interruptor de seguridad para detener la línea por seguridad, en caso de que un operador intentara abrir la puerta inferior.

El sub-ensamble denominado 0-HSS-BAS, es la plataforma que recibe la hoja con la calidad que le fue asignada al módulo correspondiente. El principal funcionamiento de este sistema es el de formar una pila de hojas de la misma calidad. Cuando inicia el proceso, la plataforma se coloca en un carro que originalmente se encuentra fuera del módulo y a través de rieles se posiciona manualmente sobre un elevador de tijera. En seguida se desplaza verticalmente mediante la acción del elevador de tijera hasta posicionarse a la altura cercana al área de trabajo del brazo. Esta altura debe mantenerse constante, por lo que a medida que se van acumulando hojas, el elevador de tijera es actuado discretamente de forma descendente. El software de control lleva registro del número de hojas que se van acumulando y cuando se llega al límite establecido (generalmente 700 hojas), el elevador baja totalmente hasta que las ruedas del carro hacen contacto con los rieles. De esta manera, el carro es recorrido manualmente hasta fuera del módulo para ser descargado y preparado para iniciar un nuevo ciclo. Por cuestión de espacio, se omite la vista de este sub-ensamble. No obstante, se puede apreciar en parte inferior derecha de la vista lateral de la figura 6.

El transportador de banda, cuyo sub-ensamble se especifica como 0-HSS-BED, tiene dos funciones: como transportador cuando se encuentra en posición horizontal, o bien, como alimentador de la estación contigua, cuando éste se inclina para entregar la hoja. Por ejemplo, si la hoja debe ser descargada en la octava estación, entonces los módulos del uno al siete se mantienen horizontales con sus bandas en movimiento $y$, cuando la hoja llega al séptimo módulo, éste se inclina y sus bandas se detienen cuando la hoja llega a la posición en que se sincroniza con el brazo del octavo módulo. En la figura 3, se muestran una vista lateral con dimensiones en milímetros y una vista isométrica que muestra el sistema de bandas por la parte inferior.
El sub-ensamble 0-HSS-GRI-0, denominado brazo sujetador, es el que tiene la función de tomar la hoja del alimentador y posicionarla en la base. Este sistema se desarrolla en la siguiente sección para presentar los conceptos que se aplican cuando se llega a la fase de detallar cada parte que contiene un sub-ensamble. La figura 4 presenta varias vistas incluyendo un detalle de la pinza sujetadora.

Al mismo tiempo que se trabaja en el diseño mecánico del sistema, se estudian los aspectos del control de los movimientos y la forma en que éstos deben llevarse a cabo. Así, se definen las entradas y salidas digitales y/o analógicas necesarias y la forma en que éstas deberán ser controladas. Para una estación del sistema se determinaron once entradas para interpretar las señales de sensores y/o interruptores y ocho salidas para actuar válvulas y/o motores. Además, dos salidas analógicas para controlar el movimiento del brazo. Con el fin de controlar un sistema de hasta 12 estaciones, se eligieron dos tarjetas electrónicas industriales con 120 canales digitales cada una (PCI-DIO-120), y una tarjeta con 16 canales analógicos (PCI-AOB-16/12). La comunicación de estas tarjetas con la computadora se logra a través de puertos PCI. El diagrama eléctrico principal del sistema se ilustra en la figura 5, en el que se introduce el concepto mediante el cual se visualiza por regiones enmarcadas con líneas de centros, el encadenamiento que se logra entre el gabinete de control principal y sus periféricos, como es el caso aquí de la consola principal 0-HSS-CP0, la consola secundaria 0-HSS-CP1 y los gabinetes satélites 0-HSS-CP2 que se encuentran instalados en cada uno de los módulos.

El ensamble general, en el que se presentan una estación y el alimentador principal, se ilustra en la figura 6. Es posible comparar esta última con el boceto que representa el concepto que se tomó como punto de partida (figura 1) y notar que algunos rasgos de las ideas principales quedan plasmadas en el resultado final.

Actualmente se encuentran en operación dos líneas de diez estaciones y una de doce estaciones en la Empresa Placage Unique, Inc., Canadá, (www.placage. com). En la figura 7 se presentan vistas similares, tanto del diseño virtual como del ensamble real de una versión de diez estaciones, mientras que en la figura 8 se ilustran dos HSS en operación.

Como referencia, se presentan en la tabla 3 el total de los elementos que se utilizaron en el diseño completo de una HSS de doce estaciones (González, 2003). Las cifras se presentan en tres rubros principales, esto es, los elementos que fueron diseñados y que llevaron un proceso de maquinado, los accesorios de diversas marcas que se adquirieron en el mercado industrial y los elementos de sujeción, siendo en promedio alrededor de 2000 componentes por módulo. 


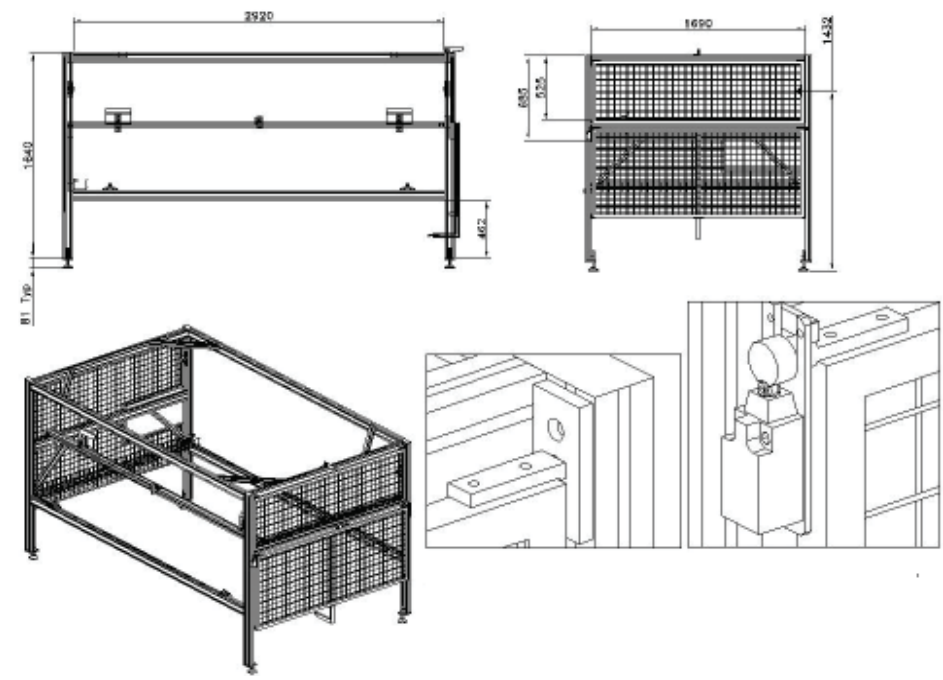

Figura 2. Subensamble de la estructura 0-HSS-FRA

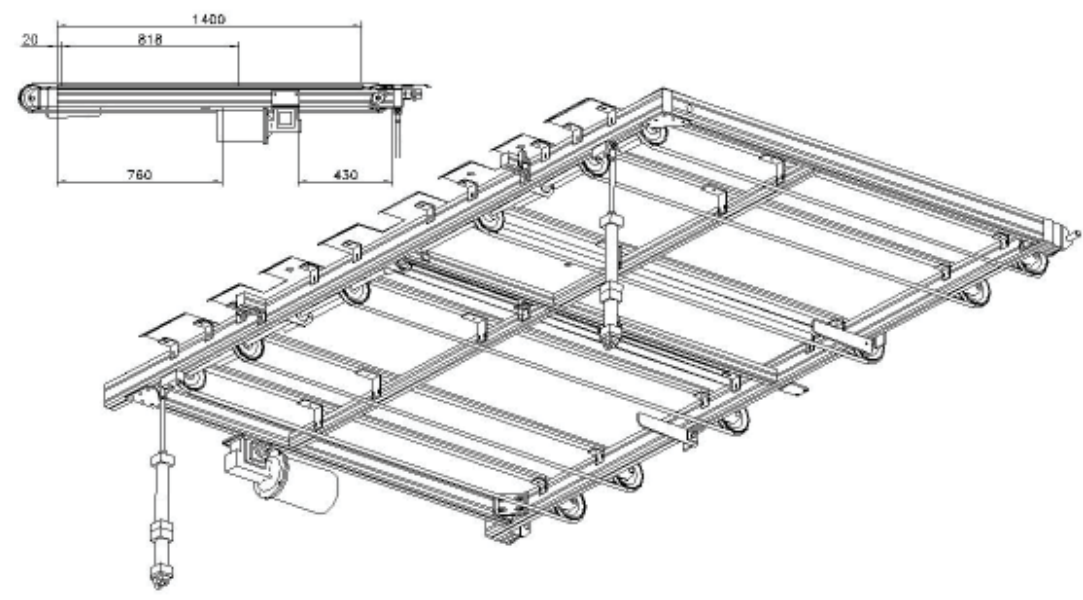

Figura 3. Sub-ensamble 0-HSS-BED

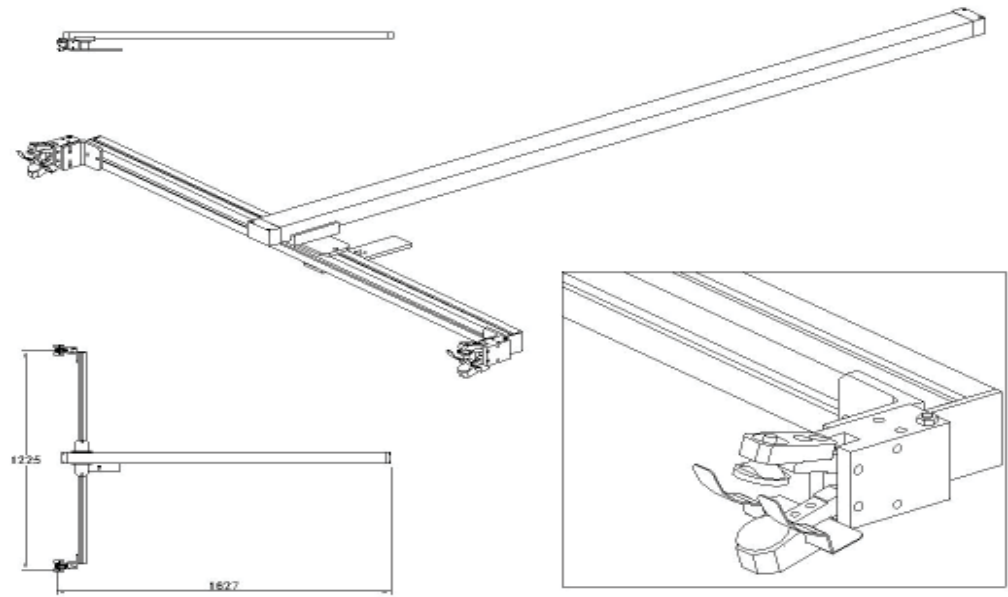

Figura 4. Sub-ensamble 0 -HSS-GRI 

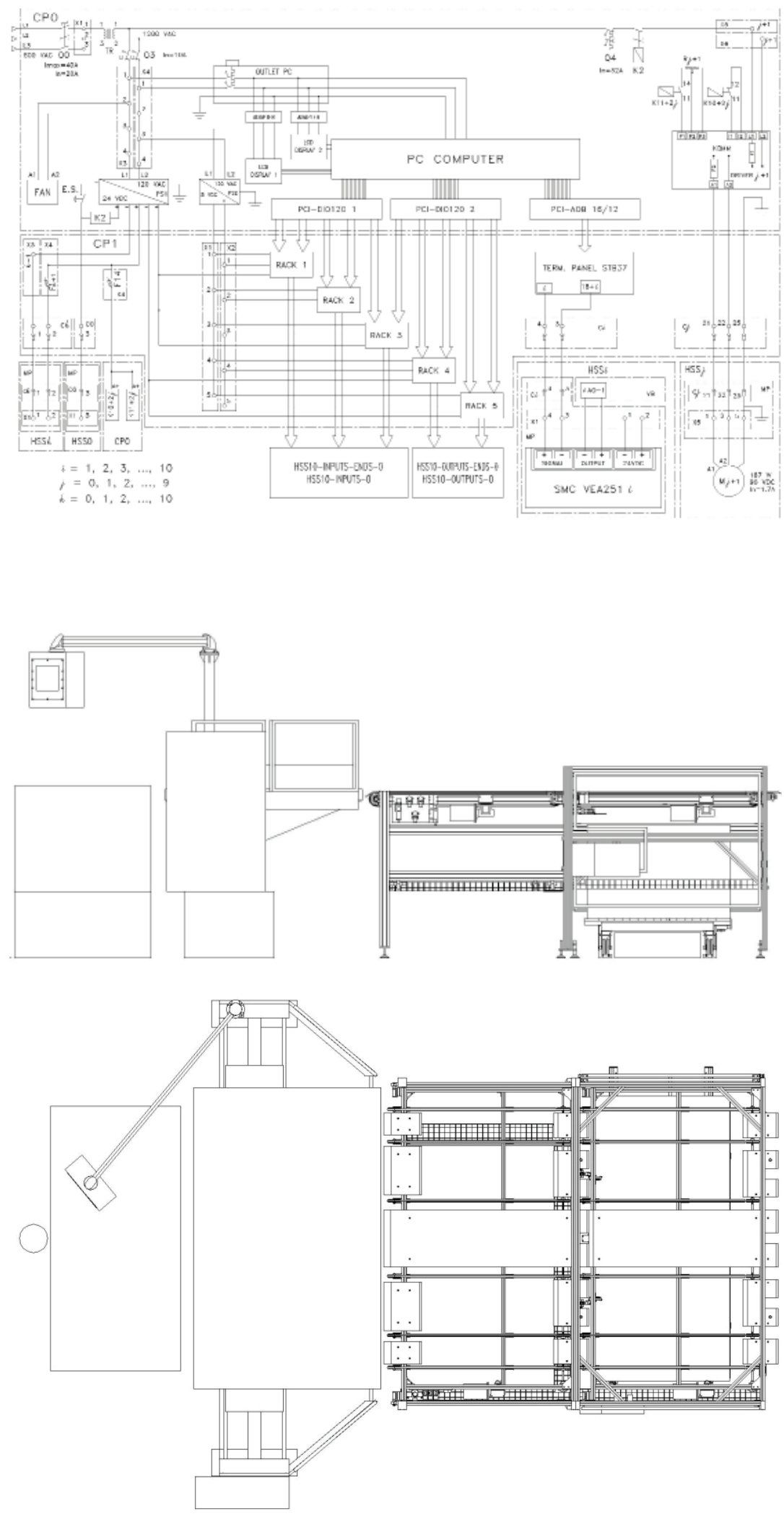

Figura 5. Diagrama Eléctrico Principal de la HSS

Figura 6. Diseño mostrando la unidad alimentadora y una estación 

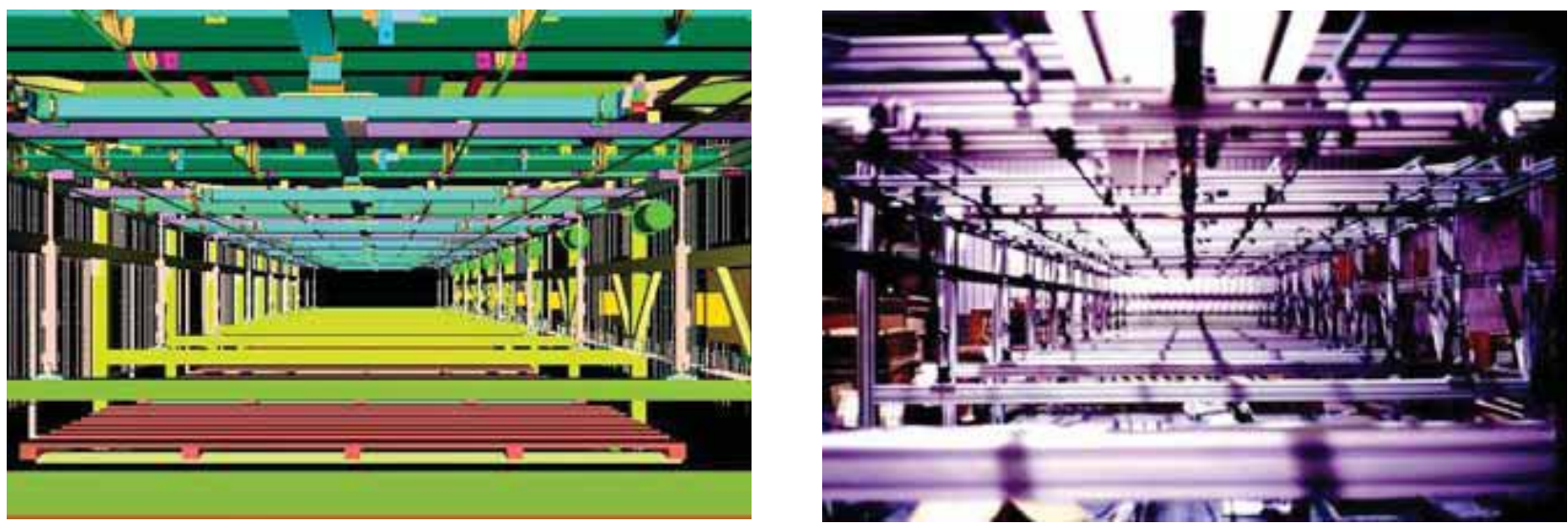

Figura 7. Prototipo virtual y ensamble final de la HSS (con autorización de Placage Unique, Inc.)

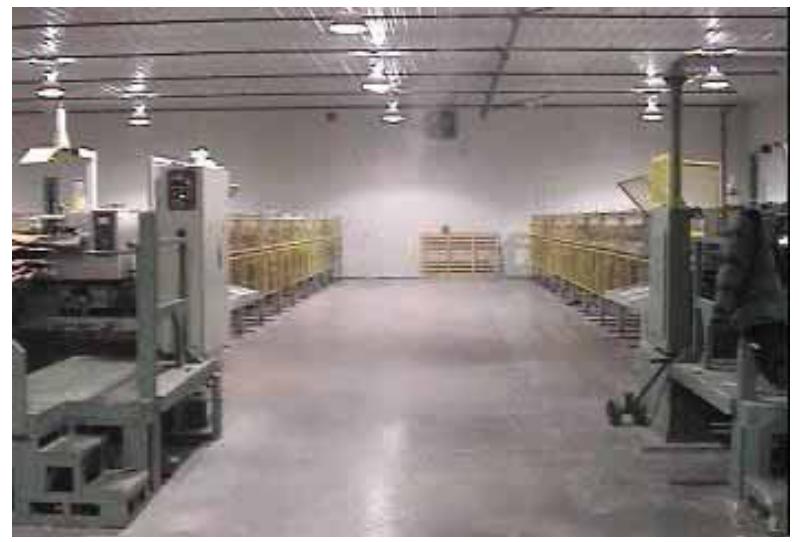

Figura 8. Dos sistemas HSS (con autorización de Placage Unique, Inc.)

Tabla 3. Elementos requeridos para ensamblar una HSS de doce estaciones

\begin{tabular}{lc}
\hline \multicolumn{1}{c}{ Rubros principales } & Cantidad \\
\hline Partes diseñadas & 2338 \\
Accesorios & 8847 \\
Sujetadores & 11830 \\
\hline Total & 23015 \\
\hline
\end{tabular}

\section{El diseño de las partes}

De la misma manera que se plantean ideas y bocetos del concepto general de un sistema, se plantean ideas y bocetos para la definición de un sub-ensamble, así como también se generan ideas y bocetos para la definición de cada parte que integra al sub-ensamble. Para ilustrar este ejercicio, se presentan a continuación los pasos que se siguieron desde el planteamiento del con- cepto, hasta la realización de las partes del sub-ensamble 0-HSS-GRI-0, denominado como brazo sujetador.

El brazo sujetador representa una componente medular de la máquina al ser el elemento que toma la hoja y la coloca sobre la pila. De esta manera, el ciclo de la máquina y consecuentemente su capacidad de producción dependen en gran medida de la velocidad con que esta tarea se realice, esto es, tomar la hoja, arrastrarla a su posición final, liberarla y regresar, quedando así preparado para tomar la siguiente. La solución que mejor cumplió con las características indicadas en la sección Planteamiento y Objetivo, fue la implementación de un pistón neumático con válvulas proporcionales reguladas con señal analógica, permitiendo así programar el perfil de velocidad óptimo tanto de avance como de retorno.

Al lado izquierdo de la figura 9 se presenta una simulación del concepto de tomar la hoja de la línea de transporte y llevarla a la pila correspondiente, mientras que en el lado derecho, se ilustra la forma en que se hicieron pruebas preliminares para determinar los rangos de la fuerza necesaria para arrastrar la hoja.

Por otra parte, una vez que se ha definido la solución sobre cómo llevar la hoja a su destino, se pasa a la definición de las partes integrantes a través de bocetos preliminares y posteriormente se comienza a definir el detalle de cada una de ellas.

Como paso siguiente, una vez definidas todas las partes a detalle, el prototipo virtual queda establecido y a través de éste se comprueba que no haya interferencia ni colisiones inesperadas en las partes móviles. Finalmente, se envían los dibujos de producción a los talleres de maquinado para la manufactura y así culminar con el ensamble de las partes correspondientes. La figura 10 muestra ambos el prototipo virtual y el real.

Una vez que el brazo se integra, se ensambla un prototipo experimental para evaluar su desempeño me- 


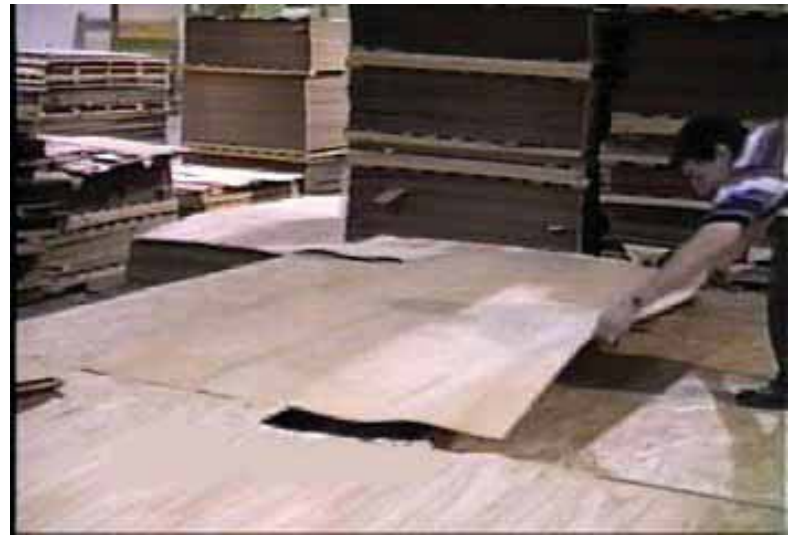

Figura 9. Conceptualización y pruebas del brazo transportador

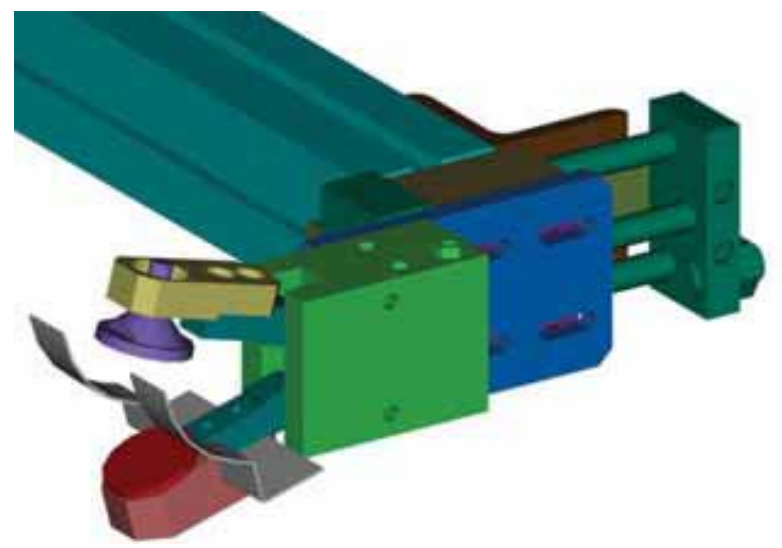

Figura 10. Prototipos virtual y real de la pinza

diante pruebas preliminares. Aquí mismo se prueban diversos algoritmos para definir la lógica del control del movimiento. Por ejemplo, se consideró como una primera instancia que el sistema de banda transportadora que sostiene el brazo se mantuviera horizontal; sin embargo, esto generaba problema de alineación de la hoja con las que ya estaban acumuladas. Después de una serie de iteraciones combinadas con sus correspondientes algoritmos de control, se determinó que la solución en la que mejor alineamiento se logra es aquella en la que el sistema de banda transportadora se inclina para que el brazo al final de su carrera quede lo más cercano posible a la pila. Por supuesto, esta solución acarrea una serie de cambios en la lógica del transporte ya que a diferencia de la primera iteración donde las bandas permanecían horizontales y podían contener hojas en transporte, ahora durante este proceso no debe haber hojas sobre las bandas del correspondiente módulo. En la figura 11 se ilustran nueve cuadros en los que se aprecia la secuencia del ciclo de arrastre de una
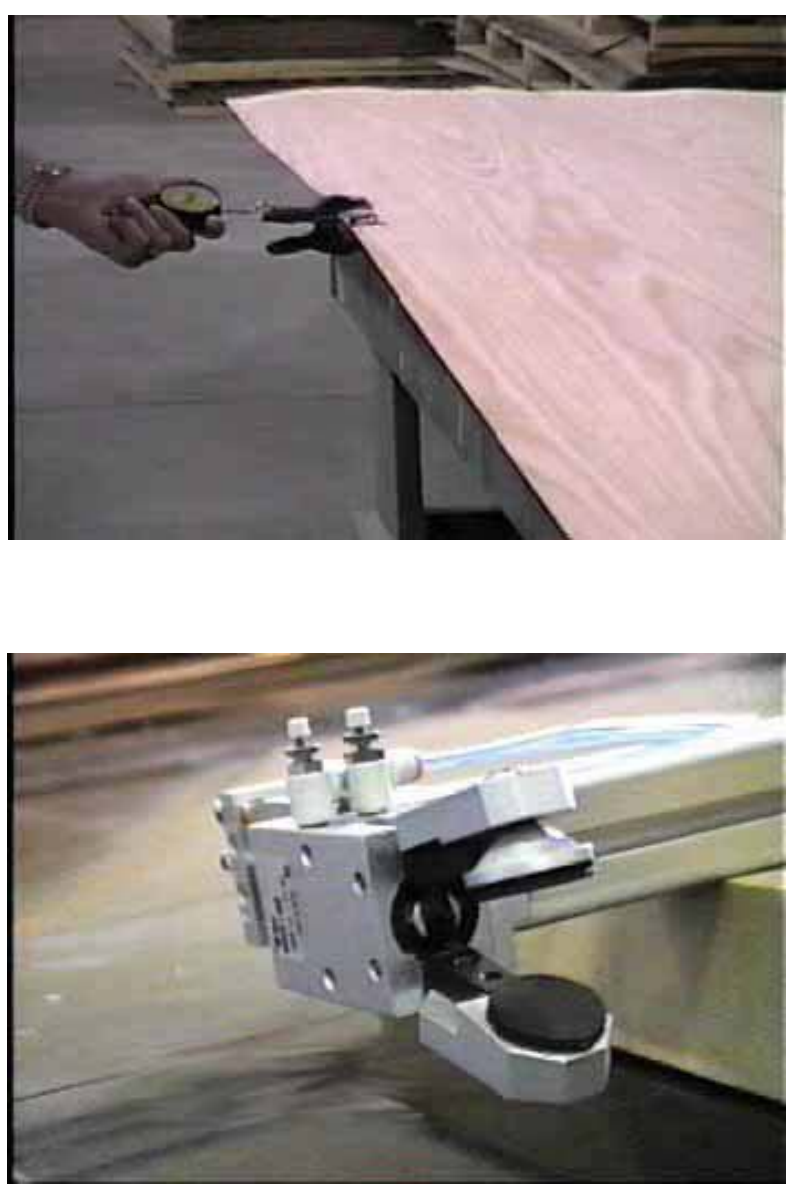

hoja. A continuación, se describe la secuencia considerando la lectura de los cuadros de izquierda a derecha y hacia abajo (el tiempo entre cuadros es de cinco décimas de segundo aproximadamente):

1. Se observa un segmento del sistema de transporte de la estación previa a la que la hoja llegará a su destino final. También se alcanza a apreciar que una hoja comienza a verse por el lado izquierdo.

2. La hoja ya ha avanzado aproximadamente $250 \mathrm{~mm}$ con respecto al cuadro anterior.

3. Se observa que el brazo ya regresó del ciclo anterior, mientras que la hoja sigue avanzando.

4. La hoja se detiene cuando los dos sensores ópticos han registrado su presencia.

5. Los dedos de la pinza se cierran y el brazo se prepara para arrastrar la hoja.

6. La hoja es arrastrada por el brazo.

7. La hoja sigue avanzando debido al arrastre del brazo.

8. La hoja está a punto de abandonar la estación. 

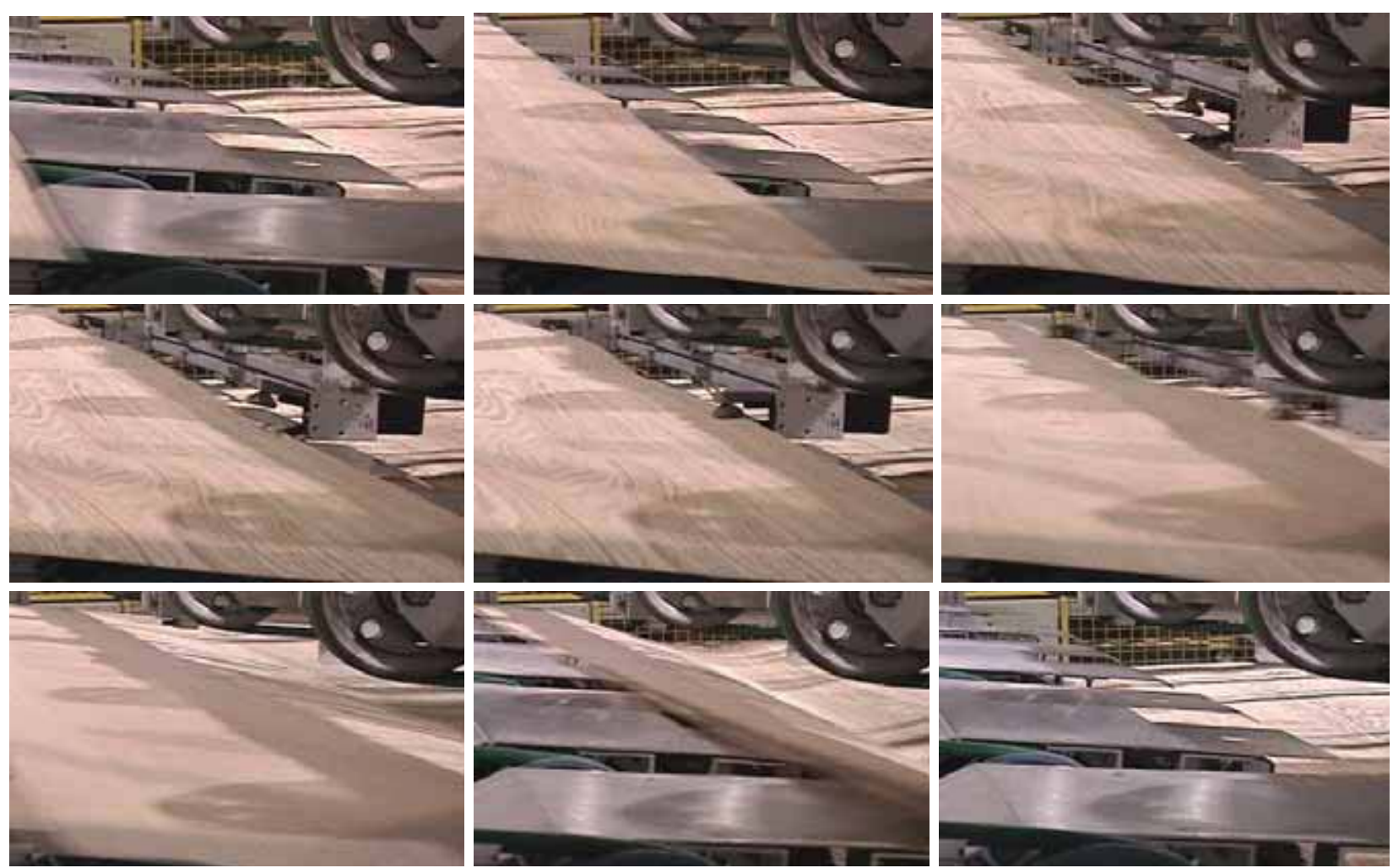

Figura 11. De izquierda a derecha y hacia abajo se presenta la secuencia del ciclo de arrastre de una hoja

9. La hoja se encuentra alineada en la pila y el brazo la libera para regresar y comenzar el ciclo nuevamente.

\section{El software de control}

Pasando ahora al aspecto de la logística del funcionamiento de la HSS, surge la pregunta: ¿Cómo se envía la hoja a su destino? Parece que una solución sencilla es poner frente al operador una estación de botones; sin embargo, si se considera que tendrá que interactuar más de 3000 veces diariamente con al menos diez botones, no es difícil darse cuenta que con esto tendrá que aumentar el grado de concentración y por consiguiente el nivel de estrés. El problema se agrava aún más cuando una calidad determinada no siempre va al mismo destino, debido a que éste puede cambiar de acuerdo a la configuración según las necesidades de producción, entonces, además de la estación de botones se torna necesario poner una tabla de direcciones, la cual sería consultada antes de presionar el botón correspondiente.

$\mathrm{Al}$ parecer, la solución más sencilla no siempre es la más indicada, ya que como en este caso, la estación de botones se convierte en una tarea complicada para el operador, ya que las probabilidades de asignar una di- rección incorrecta son muy altas. Por otra parte, el operador necesita de ambas manos para alimentar la hoja a la HSS, lo que implica que el tiempo que le lleve leer la tabla de calidades y presionar el botón correspondiente se suma al ciclo de trabajo, mermando por consiguiente, la capacidad de producción. Se requiere entonces de un análisis más profundo del problema, sin perder de vista que la premisa más importante es el hecho de facilitar la tarea al operador.

Así, tomando en cuenta que el operador debe inspeccionar la hoja para clasificarla, se concluyó que la implementación de un sistema de reconocimiento de voz es la solución idónea, ya que la sincronización de la inspección visual con voz, elimina las posibilidades de error para un operador calificado. Una vez que el operador asegura la calidad de la hoja, introduce ésta al alimentador principal al mismo tiempo que a través de un micrófono (el cual está conectado a la computadora de control) expresa en voz alta la calidad, de manera que el software de control interpreta la calidad y le asigna a la hoja la estación de destino.

Un diseño flexible abre las puertas a la diversificación. Ahora que se cuenta con el sistema de reconocimiento de voz (Lemout et al., 1997), es posible programar 
los destinos de una manera óptima y así evitar paros innecesarios en el proceso de clasificación para dar tiempo a vaciar una estación que haya llegado al límite de su capacidad.

Una vez resueltos los problemas técnicos relacionados con el funcionamiento global de la HSS, la integración de todo el sistema contiene, además de los aspectos discutidos en la sección dedicada a la integración del diseño, la realización del software y la interfaz de comunicación de la computadora con el operador de la HSS. El código fuente incluye la implementación de opciones de menú para desplegar los diálogos de comunicación para interactuar con el sistema y así poder configurar parámetros, inicializar direcciones de entradas y salidas, manipular manualmente los dispositivos de salida, monitorear señales tales como las de los sensores incluyendo aquí el sistema de reconocimiento de voz, y sobre todo, tener la interfaz gráfica que permita visualizar en línea el estado del sistema e interactuar con ésta en casos en que se tengan que hacer cambios. El código se realizó en C++ (Horton, 2006) mediante el uso de Microsoft Visual Studio.

Es importante notar que en la lógica del software de control se considera la sincronización del movimiento de las hojas sobre la línea, ya que de acuerdo a la naturaleza y flexibilidad del transporte, puede darse el caso de que varias hojas se encuentren en tránsito y más de un módulo esté recibiendo de forma simultánea, como puede apreciarse en la figura 12 donde los módulos seis y ocho (los que se ven inclinados) están descargando a los módulos siete y nueve, respectivamente.

Como resultado de este desarrollo, se han generado bibliotecas que contienen clases que han sido implementadas en aplicaciones similares (González 2005), (Collazo et al., 2008), (Rocha et al., 2008) y (González, 2008). Dichas bibliotecas actualmente se encuentran contenidas en una plataforma denominada ADEFID (González 2005).

Un ejemplo de los diálogos que se diseñaron para el sistema, se presenta en la figura 13, el cual despliega un centro virtual de botones para activar manualmente los dispositivos que componen una estación. Este diálogo también permite configurar el perfil de velocidades del movimiento del pistón del brazo.

Por otra parte, se presenta en la figura 14, la interfaz gráfica que se despliega en la consola principal del sistema. Ésta permite visualizar interactivamente el desempeño del sistema y su estado actual. Se puede apreciar que en el instante mostrado, existen 4 hojas de madera de cerezo en tránsito, dirigiéndose a la estación nueve, la cual está recibiendo la calidad "B No Sap" y que cuenta ya con 680 hojas en la pila. Además, el ope- rador acaba de ingresar una hoja de calidad "A Sap", la cual se dirige a la estación cuatro.

\section{La eficiencia}

Un estudio de productividad se realizó con base a los eventos que generan tiempos muertos y que se presentan durante una jornada de 8 horas, entre los más relevantes se pueden mencionar: el no alineamiento de una hoja, en este caso el ciclo aumenta por tomar más tiempo para que la hoja se enderece automáticamente al llegar a las pinzas del brazo; intervención del operador en alguna estación por razones inevitables, como el caso del desgarramiento de una hoja defectuosa o el desprendimiento de una unión mal pegada; o bien, el cambio de una pila, cuando ésta se llena. Dichos eventos se consideran en el estudio para hacer un análisis de la eficiencia del sistema (González, 2004). Como resultado de éste, se determinó que la producción esperada está dada por la siguiente ecuación,

$P=\eta_{S} \eta_{O} C$

donde,

$P=$ producción esperada (hojas /turno),

$C=T / \tau$, capacidad de producción (hojas/turno),

$T=$ duración del turno (segundos/turno),

$\tau=$ ciclo promedio de la HSS (segundos/hoja),

$\eta_{S}=$ eficiencia del sistema,

$\eta_{O}=$ eficiencia del operador.

La eficiencia del sistema se obtiene de la siguiente ecuacion,

$\eta_{S}=1-\frac{1}{\tau} \sum_{i=1}^{n} \frac{t_{i}}{\alpha_{i}}$

donde,

$t_{i}=$ tiempo requerido para eliminar el $i$-ésimo evento (segundos/evento),

$\alpha_{i}=$ número de hojas alimentadas antes de que ocurra el $i$-ésimo evento (hojas/evento).

$n=$ número posible de eventos durante la producción.

Pruebas realizadas reportaron que el ciclo óptimo de la HSS por cada estación es de 5.5 segundos/hoja, ya que al reducir este valor, la eficiencia del sistema comienza a decaer. Por otra parte, con esto se cumple el objetivo de que la capacidad de producción máquina-operador depende solamente del tiempo que la lleve al operador 

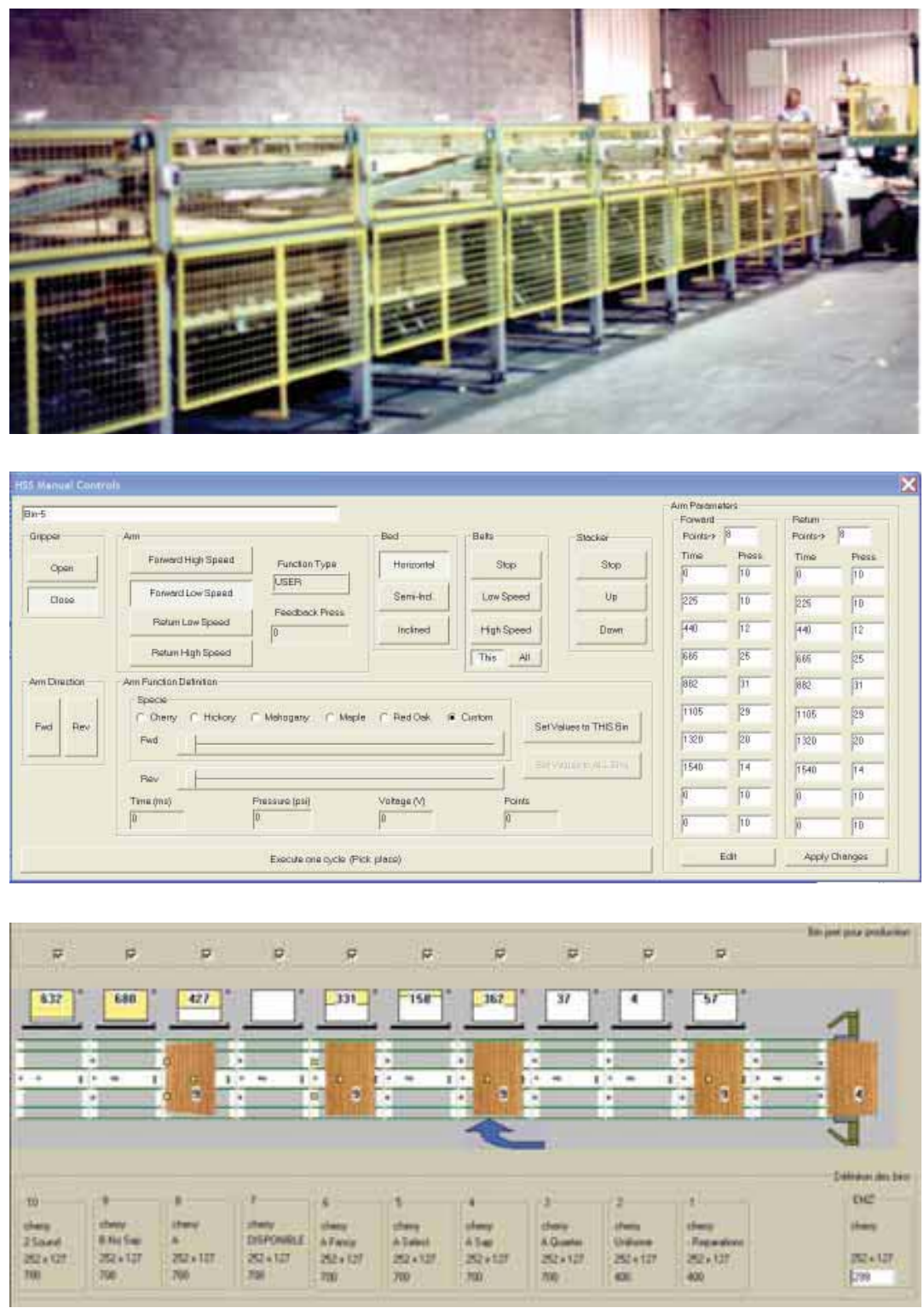

Figura 12. HSS en operación (con autorización de Placage Unique, Inc.)

Figura 13. Centro virtual de botones para actuar los dispositivos de una estación

Figura 14. Interfaz gráfica de un sistema de diez estaciones definir la calidad de la hoja, el cual, como se mencionó previamente, es de seis a ocho segundos.

\section{Conclusiones}

En este trabajo se presentaron los procedimientos de diseño en mecatrónica como una búsqueda constante del conocimiento a través de la formación teórico-práctica integral para la creación de un producto mediante la presentación de un caso de estudio. Durante el desarrollo del caso de estudio se estableció que cuando se desea diseñar un dispositivo para resolver un problema, es importante que se definan claramente tanto el objetivo como el planteamiento del problema aunado con los requisitos que se deben cubrir. Más aún, los procedimientos descritos representan un modelo para el desarrollo de otros sistemas.

Se estableció la importancia de aterrizar ideas generales de la solución en bocetos durante la etapa de conceptualización y así definir la mejor opción para tomarla como punto de partida, basando la decisión en la opción que mejor satisfaga los requisitos y características establecidos. Durante este proceso se observó que las probabilidades de éxito en la definición preliminar de la solución se incrementan en la medida en que se tenga un mayor conocimiento de las disciplinas involu- 
cradas en el problema de diseño. En el caso de estudio presentado aquí, se combinaron la ingeniería mecánica, ingeniería industrial, ingeniería en sistemas computacionales e ingeniería electrónica.

Se discutió la relevancia de generar prototipos virtuales mediante el uso de herramientas de CAD durante el proceso de la integración del diseño. La formación de un prototipo virtual requiere de la generación de una gran cantidad de modelos sólidos de cada una de las partes que intervienen, cuyas dimensiones generalmente cambian parcial o totalmente conforme se van ensamblando en un dibujo de montaje global. Se estableció una metodología de nombrar las partes y/o subensambles, para facilitar no solo su identificación sino también su seguimiento durante el proceso de evolución y/o actualización.

También se estableció que una vez que el diseño se plasma en el prototipo real y se efectúan pruebas de su desempeño para compararlo nuevamente con los requisitos originales planteados, es deseable que los resultados de estas pruebas los satisfagan, y en este sentido, se plasmó la importancia que durante el proceso de la generación del prototipo virtual, se tomen en consideración dichos requisitos.

Finalmente, la experiencia del diseñador juega un papel importante en todo el proceso, ya que en la medida que ésta se acumule y se combine con la actualización constante del conocimiento científico y tecnológico del correspondiente campo de aplicación, las probabilidades de que surjan imprevistos no deseados se irán minimizando en nuevos diseños, y así, las revisiones o cambios en los correspondientes productos finales se visualizarán en el sentido de su perfeccionamiento y/o mejoramiento, para brindar un mejor servicio en concordancia con el fin para el que éstos se crean.

\section{Agradecimientos}

El autor, como responsable directo del caso de estudio aquí presentado y quien lo desarrolló, desde el planteamiento del boceto hasta su puesta en marcha durante su estancia como director del Departamento de Investigación y Desarrollo de la Empresa Placage Unique, Inc. Canadá, agradece a Terry Gogarty, presidente de dicha empresa, por su confianza y decidida participación con recursos financieros y humanos para la ejecución del caso de estudio aquí presentado. También reconoce la colaboración de Emmanuel Maz, quien participó en el desarrollo del software de control. Al CONACyT, a través del Sistema Nacional de Investigadores, por el apoyo otorgado para la realización de este artículo.

\section{Referencias}

BDI Bearing, Power Transmission, Industrial and Safety and Fluid Power [en línea]. Quebec. BDI, 2007 [fecha de consulta 4 marzo 2007]. Disponible en: http://www.bdi-canada.com/products_mat_handling.php

Collazo-Barrientos J.C., Plascencia-Mora H. y González-Palacios M.A. Sistema electromecánico de corte de EPS en 2D computarizado. 14 Congreso Internacional de la SOMIM. 17-19 de Septiembre, 2008. Puebla. México.

Emerson Industrial Automation, Morse Catalog [en línea]. Kentucky. Emerson Power Transmission, 2002 [fecha de consulta 27 mayo 2006]. Disponible en: http://www.emerson-ept.com

Fabory Masters in Fasteners [en línea]. Ontario. Fabory, 2009 [fecha de consulta 20 mayo 2008]. Disponible en: http://www. faborymetrican.com

González-Barbosa. Síntesis, simulación y control de posición de manipuladores seriales no redundantes. (Maestría en ingeniería mecánica). México. Universidad de Guanajuato, Facultad de Ingeniería, Salamanca, Gto., 2008. 120p.

González-Palacios M.A. 12 Bin Horizontal Sorting System. México: Integración de Procesos Industriales. 2003.

González-Palacios M.A., Spliced Veneer Integrated System. Parts I-VII. México: Integración de Procesos Industriales. 2005.

González-Palacios M.A. ADEFID: Advanced Engineering SoFtware for Industrial Development. México: Integración de Procesos Industriales. 2005.

González-Palacios M.A. Horizontal Sorting System, a Productivity Point of View. México: Integración de Procesos Industriales. 2004.

Horton I. Beginning Visual C++ 2005. UK. Wrox Press. 2006. 1224p.

Igus Energy Chain Systems [en línea]. Eeast Providence. Igus Inc., 2007 [fecha de consulta 4 Junio 2007]. Disponible en: http://www.igus.com

$\mathrm{KB}$ a Complete Line of Motor Drives [en línea]. Florida. KB Electronics Inc., 2007 [fecha de consulta 5 abril 2007]. Disponible en: http://www.kbelectronics.com

Kontron Computer-on-modules [en línea]. Quebec. Kontron, 2010 [fecha de consulta 2 marzo 2007]. Disponible en: http://www. kontron.com

Lernout and Hauspie Voice Xpress Plus [en línea]. 21st Century Eloquence, 1997 [fecha de consulta 10 octubre 2007]. Disponible en: http://www.voicerecognition.com/1998/products/lernout_hauspie/voicexpressplus.html

NTN Bearing Corporation of Canada [en línea]. Quebec. NTN [fecha de consulta 14 julio 2006]. Disponible en: http://www.ntn.ca

Oberg E., Jones F.D., Ryffel H.H., McCauley C.J., Heald R.M. Machinery's Hand-Book. 28/e. New York. Industrial Press Inc. 2008. 2704p.

Omron Sensing Solutions [en línea]. Chicago. Omron Electronics, 2006 [fecha de consulta 9 agosto 2006]. Disponible en: http://www.omron.com

Omura G. Mastering AutoCAD 2010 and AutoCAD LT 2010. $1^{\text {st }}$ ed. Indiana. Wiley Publishing, Inc. 2009. Pp. 955-990. 
Panduit Cable Ties and Wiring Accessories [en línea]. Tinley Park. Panduit Corp., 1998 [fecha de consulta 20 noviembre 2007]. Disponible en: http://www.panduit.com

Parker Electromechanical Actuator Products, Catalog AU03-18942/US [en línea]. Cleveland. Parker Hannifin Corporation, 2007 [fecha de consulta 15 febrero 2008]. Disponible en: http:// www.parkermotion.com

Polyfab Catalogue des Produits et Services [en línea]. Montreal. Plastique Polyfab, 1998 [fecha de consulta 12 abril 2006]. Disponible en: http://www.plastiquepolyfab.com

Rittal Industrial Enclosures [en línea]. Urbana. Rittal Corporation, 2008 [fecha de consulta 30 enero 2008]. Disponible en: http://www.rittal-corp.com

Rocha-Aguilera G., González-Palacios M.A., Colín-Venegas J. y Aguilera-Cortés L.A. Simulación en ADEFID del movimiento vibratorio de una cuerda. 14 Congreso Internacional de la SOMIM. 17-19 de Septiembre, 2008. Puebla, México.
SMC Corporation of America, Pneumatic Automation Products [en línea]. Noblesville. SMC Corporation of America, 2000 [fecha de consulta 17 octubre 2007]. Disponible en: http://www. smcusa.com

Turk Molded Cordsets and Wiring Devices [en línea]. Minneapolis. Turk Inc., 1995 [fecha de consulta 2 mayo 2007]. Disponible en: http://www.turck.com

US Motors, DC Motors and Drives [en línea]. Orange. US Motors, 1995[fecha de consulta 15 agosto 2007]. Disponible en: http:// www.usmotors.com

Wieland Electrical Connections [en línea]. Burgaw. Wieland Inc., 1999 [fecha de consulta 23 febrero 2008]. Disponible en: http:// www.wielandinc.com

Winsmith Worm Gear Speed Reducers [en línea]. Springville. Peerless-Winsmith, Inc., 1997 [fecha de consulta 27 enero 2008]. Disponible en: http://www.winsmith.com

\section{Semblanza del autor}

Max Antonio González-Palacios. Obtuvo la licenciatura en ingeniería mecánica y eléctrica en 1986 por la Universidad Iberoamericana-León. Asimismo, la maestría en ingeniería por la Universidad de Guanajuato y el doctorado en 1992 por la Universidad McGill, Montreal, donde completó un Posdoctorado en 1993. En la industria, de 1997 a 2000 dirigió el Departamento de Investigación y Desarrollo de Placage Unique, Inc., Québec. Desde el 2000, es director de IPI (Integración de Procesos Industriales), donde ha desarrollado diversos proyectos de investigación aplicada. Actualmente es profesor titular de la División de Ingenierías, Campus IrapuatoSalamanca de la Universidad de Guanajuato. 